\title{
Structural evolution and flip-flop recombination of chloroplast DNA in the fern genus Osmunda
}

\author{
Diana B. Stein ${ }^{1}$, Jeffrey D. Palmer ${ }^{2}$, and William F. Thompson ${ }^{3}$ \\ 1 Department of Biological Sciences, Mount Holyoke College, South Hadley, MA 01075, USA \\ 2 Department of Biology, University of Michigan, Ann Arbor, MI 48109, USA \\ 3 Carnegie Institution of Washington, 290 Panama Street, Stanford, CA 94305, USA
}

Summary. The evolution and recombination of chloroplast genome structure in the fern genus Osmunda were studied by comparative restriction site mapping and filter hybridization of chloroplast DNAs (cpDNAs) from three species - $O$. cinnamomea, $O$. claytoniana and $O$. regalis. The three $144 \mathrm{~kb}$ circular genomes were found to be colinear in organization, indicating that no major inversions or transpositions had occurred during the approximately 70 million years since their radiation from a common ancestor. Although overall size and sequence arrangement are highly conserved in the three genomes, they differ by an extensive series of small deletions and insertions, ranging in size from $50 \mathrm{bp}$ to $350 \mathrm{bp}$ and scattered more or less at random throughout the circular chromosomes. All three chloroplast genomes contain a large inverted repeat of approximately $10 \mathrm{~kb}$ in size. However, hybridizations using cloned fragments from the $O$. cinnamomea and $O$. regalis genomes revealed the absence of any dispersed repeats in at least $50 \%$ of the genome. Analysis with restriction enzymes that fail to cleave the $10 \mathrm{~kb}$ inverted repeat indicated that each of the three fern chloroplast genomes exists as an equimolar population of two isomeric circles differing only in the relative orientation of their two single copy regions. These two inversion isomers are inferred to result from high frequency intramolecular recombination between paired inverted repeat segments. In all aspects of their general organization, recombinational heterogeneity, and extent of structural rearrangement and length mutation, these fern chloroplast genomes resemble very closely the chloroplast genomes of most angiosperms.

Key words: Inverted repeat - Colinearity - Flip-flop recombination - Fern chloroplast DNA

Offprint requests to: J. D. Palmer

\section{Introduction}

Detailed information about evolutionary change in chloroplast genome structure is presently available for only two groups of plants - flowering plants and the green algal genus Chlamydomonas. Comparisons among chloroplast DNAs (cpDNAs) from over 200 species of angiosperms indicate that major structural changes occur only very rarely (reviewed in Whitfeld and Bottomley 1983; Gillham et al. 1985; Palmer 1985a, 1985b). Crosshybridization and gene mapping studies have revealed that the majority of angiosperm cpDNAs have the same gene order and arrangement, and that most of the exceptional genomes differ from the norm by only one or two inversions (Fluhr and Edelman 1981; Palmer and Thompson 1982; Palmer et al. 1983a, 1983b; de Heij et al. 1983; Herrmann et al. 1983; Mubumbila et al. 1984, 1985). Genome size is also highly conserved among angiosperm cpDNAs. Most of these genomes vary in size only from $135 \mathrm{~kb}$ to $160 \mathrm{~kb}$ (reviewed in Bohnert et al. 1982; Crouse et al. 1985; Gillham et al. 1985) and essentially all the length mutations detected in sequencing and restriction mapping studies of closely related species are short, from one or two bp to $1,000 \mathrm{bp}$ (e.g. Gordon et al. 1982; Takaiwa and Sugiura 1982; Bowman et al. 1983; Palmer et al. 1983b, 1985a; Zurawski et al. 1984; Salts et al. 1984).

Rates of cpDNA structural evolution appear to be higher in Chlamydomonas than among flowering plants. The chloroplast genomes of four Chlamydomonas species vary in size from $195 \mathrm{~kb}$ to $292 \mathrm{~kb}$ and in sequence complexity by $78 \mathrm{~kb}$, over twice the range $(35 \mathrm{~kb})$ found among all of the more than 200 angiosperm genomes examined (Rochaix 1978; Palmer et al. 1985b; Lemieux et al. 1985a, 1985b). Moreover, small deletions and additions appear to occur at a higher frequency relative to base substitutions in Chlamydomonas cpDNA than in 


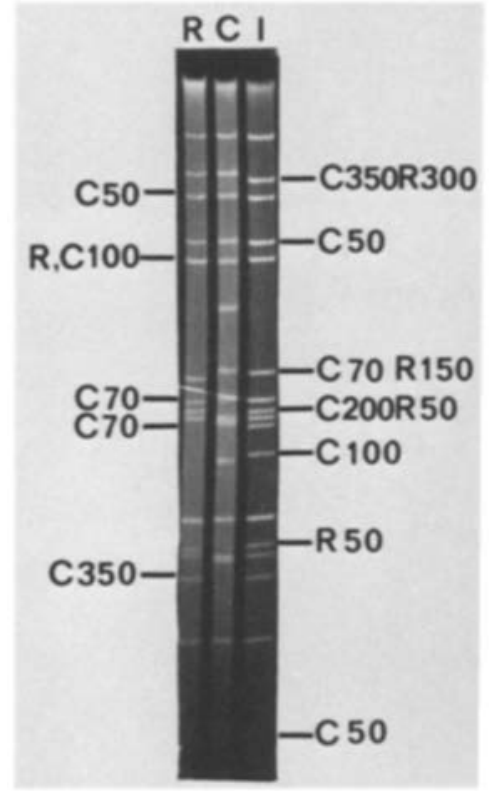

Fig. 1. Comparison of PvulI restriction fragment patterns of Osmunda cpDNAs. CpDNAs from Osmunda regalis (R), O. claytoniana $(\mathrm{C})$, and $O$. cinnamomea (I) were digested with PvuII and the resulting fragments were separated by electrophoresis in a $0.7 \%$ agarose gel. Numbers next to various bands refer to size differences (in base pairs) between the cinnamomea and either the claytoniana (C) and/or regalis ( $\mathrm{R}$ ) bands. Assignment of differences in fragment size is based on this gel as well as two other gels containing the same digests

angiosperm cpDNA (Palmer et al. 1985b) Finally, two distantly related Chlamydomonas species differ in gene order by an extensive series of sequence rearrangements (Lemieux and Lemieux 1985). This suggests that rates of inversion and transposition may be significantly higher in Chlamydomonas cpDNA, although other explanations are possible (Lemieux and Lemieux 1985) and more comparisions desirable.

This disparity in rates of structural evolution among these two groups of chloroplast genomes makes one wonder about genome evolution in other groups of plants. In particular, how do cpDNAs vary in structure and organization among non-angiospermous land plants, such as gymnosperms, ferns and bryophytes, which share a common ancestry with angiosperms but which comprise distinct phyletic lines separated for hundreds of millions of years? Thus far, there have been no comparisons of chloroplast genome evolution within specific groups of non-angiospermous land plants, although the cpDNAs of the few ferns and bryophytes that have been examined do show some general similarities to angiosperm $\mathrm{cpDNAs}$ in overall size and certain rudimentary organizational features (Herrmann et al. 1980; Palmer and Stein 1982; Ohyama et al. 1983). In this report, we make the first detailed comparative study of the structure and evolution of cpDNA within a specific group of non-angiosper- mous land plants. We show that three chloroplast genomes from the fern genus Osmunda, which diverged from each other on the order of 70 million years ago (Miller 1967, 1971), are essentially identical in linear order and size, differing in structure only by a series of small deletions and additions.

One structural feature which is common to almost all green algal and angiosperm cpDNAs (reviewed in Crouse et al. 1985; Palmer 1985a, 1985b), as well as those of the fern Osmunda cinnamomea (Palmer and Stein 1982) and the bryophyte Marchantia polymorpha (Ohyama et al. 1983), is a large (10-76 kb) inverted repeat encoding a complete set of chloroplast ribosomal RNAs. In those few angiosperm and green algal genomes for which restriction enzymes have been found that fail to cleave within this inverted repeat it has been possible to show that the genome exists as a mixture of two inversion isomers differing in the relative polarity of their single copy regions (Palmer 1983; Mubumbila et al. 1983; Palmer et al. 1984, 1985b; Aldrich et al. 1985), presumably as the result of intramolecular recombination between inverted repeat segments. Here we show that all three Osmunda species examined contain a $10 \mathrm{~kb}$, rRNA-encoding inverted repeat that confers the same sort of inversional heterogeneity to the chloroplast genome.

\section{Materials and methods}

cpDNA was prepared as described (Palmer and Stein 1982). Plasmid clones containing PstI fragments of the Osmunda cinnamomea and $O$. regalis chloroplast genomes were prepared by $\mathrm{V}$. James and G. Bennett. PstI-digested total cpDNA was ligated with PstI-digested pUC8 or pUC9 (Vieira and Messing 1982), transformed into $E$. coli strain JM83, and recombinant white colonies were selected on ampicillin/X-gal plates. The clone banks were characterized by sizing PstI-digested plasmid DNAs isolated from one $\mathrm{ml}$ of bacterial culture by the alkaline extraction procedure of Birnboim and Doly (1979). Restriction endonuclease digestions, agarose gel electrophoresis, bidirectional transfers of DNA fragments from agarose gels to nitrocellulose filters, labeling of recombinant plasmids by nick-translation, and filter hybridizations were performed as previously described (Palmer 1982, 1985c). All filters were washed in $2 \times \operatorname{SSC}(0.3 \mathrm{M}$ $\mathrm{NaCl} / 30 \mathrm{mM}$ trisodium citrate) and $0.5 \%$ SDS at $65^{\circ}$ prior to autoradiography.

\section{Results}

\section{Structural comparisons}

We compared the structure of the chloroplast genomes of three species of the fern genus Osmuda, which are thought to have been separated for at least 70 million years (Miller 1967, 1971; Stein et al. 1979). Initially we compared purified cpDNAs from the three species, $O$. cinnamomea, $O$. claytoniana, and $O$. regalis, by agarose gel electropho- 


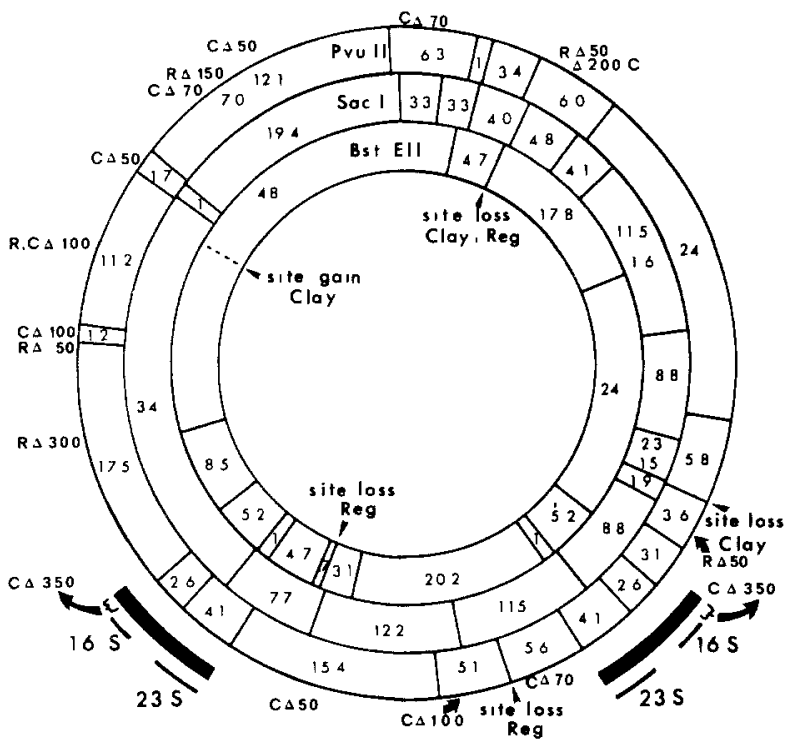

Fig. 2. Comparison of physical maps of cpDNA from three species of Osmunda. Locations and sizes of PvuII, SacI and BstEII restriction fragments are indicated for the reference chloroplast genome from $O$. cinnamomea (data taken from Palmer and Stein 1982). Loss or gain of restriction sites in the BstEII and PvulI maps only, is shown for cpDNAs from $O$, regalis $(\mathrm{Reg})$ and $O$. claytoniana (Clay) and is based on hybridization of each of the 19 PvulI fragments from $O$. cinnamomea to filter blots containing BstEII and PvulI fragments of the other two species. Differences in fragment sizes (in base pairs) are given for $O$. claytoniana (C) and $O$. regalis $(\mathrm{R})$ with reference to $O$. cinnamomea PvuII fragments (Fig. 1). Note that only one of the two genome orientations is shown (see text and Fig. 5).

resis of fragments produced by some 25 restriction endonucleases that have 6-bp recognition sites (data not shown). Not surprisingly, given the long period of separation of the three species, their cpDNA restriction patterns showed a large number of differences and relatively few similarities with most of the enzymes surveyed. However, one enzyme in particular, PvuII, produced strikingly similar fragment patterns for all three cpDNAs (Fig. 1).

In order to determine the overall arrangement of homologous sequence elements in the three genomes we hybridized each of the 19 nick-translated, gel-purified (Palmer 1982) PvuII fragments from O. cinnamomea, for which a complete restriction map has already been published (Palmer and Stein 1982), to nitrocellulose filters containing restriction fragments of all three genomes produced by PvuII, BstEII, SacI, and PstI. The results of this hybridization study are summarized in Fig. 2 for PvuII and BstEII, the two enzymes whose recognition sites have been least altered among the three cpDNAs. The three genomes were found to be identical in linear sequence order and extremely similar in absolute sequence arrangement, differing only by a series of small deletions and additions of between $50 \mathrm{bp}$ and $350 \mathrm{bp}$ in size (Fig. 2). These length mutations are best seen by comparing the PvuII patterns (Fig. 1) and maps (Fig. 2), in which $O$. claytoniana and $O$. regalis cpDNAs are seen to differ from that of the reference species, $O$. cinnamomea, by the loss of only a single Prull site each. The Pvull patterns (Fig. 1) reveal a large number of small mobility differences among fragments that map to the same position (Fig. 2). Assuming a random distribution of base substitutions, statistical considerations suggest that most of these differences must reflect small deletions and additions within fragments, rather than base substitutions that create new restriction sites near the ends of fragments. Note that the length mutations are scattered more or less randomly throughout the genome.

\section{Repeated sequences}

The only repeated element in the three Osmunda cpDNAs revealed by the restriction mapping and cross-hybridization studies is a large inverted repeat, approx. $10 \mathrm{~kb}$ in size, that encodes a complete set of ribosomal RNA genes (Fig. 2; Palmer and Stein 1982). As in angiosperms, the Osmunda inverted repeat is situated asymmetrically, with the same orientation of the rRNA operon relative to the small and large single copy regions. However, as previously noted (Palmer and Stein 1982), the Osmunda repeat is substantially smaller than the vast majority of angiosperm repeats, which are generally between $20 \mathrm{~kb}$ and $30 \mathrm{~kb}$ in size (Bohnert et al. 1982; Crouse et al. 1985). A single large insertion of $350 \mathrm{bp}$ has occurred symmetrically in both segments of the inverted repeat in $O$. claytoniana relative to the other two species (Fig. 2 ). The symmetric nature of this inverted repeat "insertion" is consistent with a large body of data derived from comparisons of angiosperm and green algal cpDNAs indicating that some sort of copy-correction mechanism maintains sequence identity between the repeat elements present within a given cpDNA chromosome (reviewed in Gillham et al. 1985; Palmer 1985a, 1985b).

Although no other repeats were revealed by the hybridization studies, this conclusion is necessarily limited by the fact that the probes consisted of uncloned PvuII fragments purified from digests of total cpDNA and which always showed a low level of cross-contamination with closely migrating PvulI fragments. In order to detect dispersed repeated sequences with greater sensitivity we hybridized various cloned PstI fragments of $O$. regalis and $O$. cinnamomea cpDNAs to filter blots containing PstI fragments of the parent genomes. This self-hybridization analysis was performed with a single $O$. regalis clone containing a $9.7 \mathrm{~kb}$ fragment that is located primarily within the small single copy region, and with seven $O$. cinnamomea clones that cover 57.8 $\mathrm{kb}$ of the large single copy region (Figs. 3,5 ). None of 


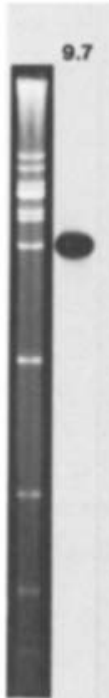

A

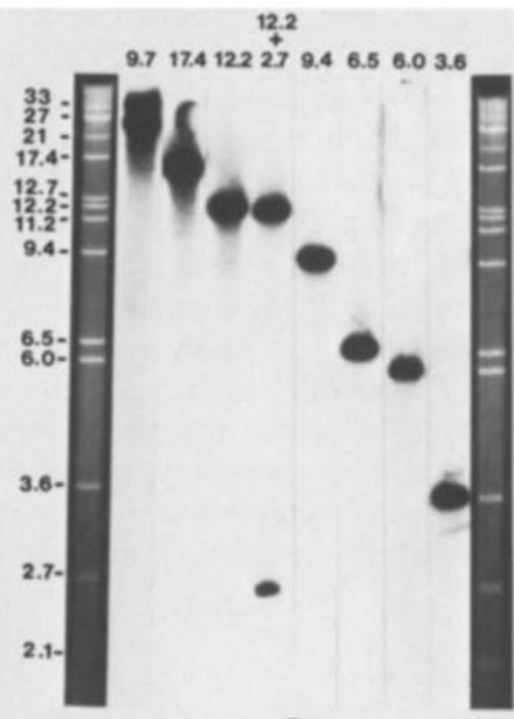

B
Fig. 3. Absence of dispersed repeated sequences in the Osmunda chloroplast genome. Panel A: Hybridization of a clone containing a $9.7 \mathrm{~kb}$ PstI fragment from $O$. regalis to a filter containing PstI fragments of $O$. regalis cpDNA that had been separated on a $0.7 \%$ agarose gel. Panel $B$ : Eight identical filter strips containing Pstl restriction fragments of $O$. cinnamomea cpDNA that had been separated on a $0.7 \%$ agarose gel were hybridized with each of seven plasmid clones containing $O$. cinnamomea Pstl restriction fragments (sizes in $\mathrm{kb}$ of the Pstl inserts are given above each filter strip) and with a clone containing the $9.7 \mathrm{~kb}$ PstI fragment from $O$. regalis these clones hybridized to any PstI fragments except for the one (s) contained within the clone itself (Fig. 3 and in a 5 -fold longer autoradiographic exposure of the filters used to produce Fig. 3). This analysis therefore rules out the presence of dispersed repeated sequences within the $50 \%$ of the genome (in terms of sequence complexity) covered by the clones used. It assumes any repeats would be of sufficient size and sequence homology as to be detectable under our conditions of hybridization (washes were performed in $2 \times \mathrm{SSC}$ at $65^{\circ}$ ) and autoradiography. It is still possible that short repeated sequences are present, either clustered within an individual PstI fragment or distributed among the PstI fragments not used as hybridization probes.

\section{Flip-flop recombination}

The cross-hybridization mapping experiments described in the first section revealed that the enzyme PstI does not cleave within the inverted repeat of $O$. cinnamomea and that SacI fails to cleave within the inverted repeats of $O$. claytoniana and $O$. regalis (data not shown). Resolution of these enzyme digests in a $0.5 \%$ agarose gel run at low voltage for $24 \mathrm{~h}$ revealed the presence of large, approximately half-molar bands for each cpDNA (Fig. 4). For O. cinnamomea there are two half-molar PstI fragments, of $21 \mathrm{~kb}$ and $33 \mathrm{~kb}$, and the sum of all the PstI fragments is approx. $27 \mathrm{~kb}$ larger than the genome size
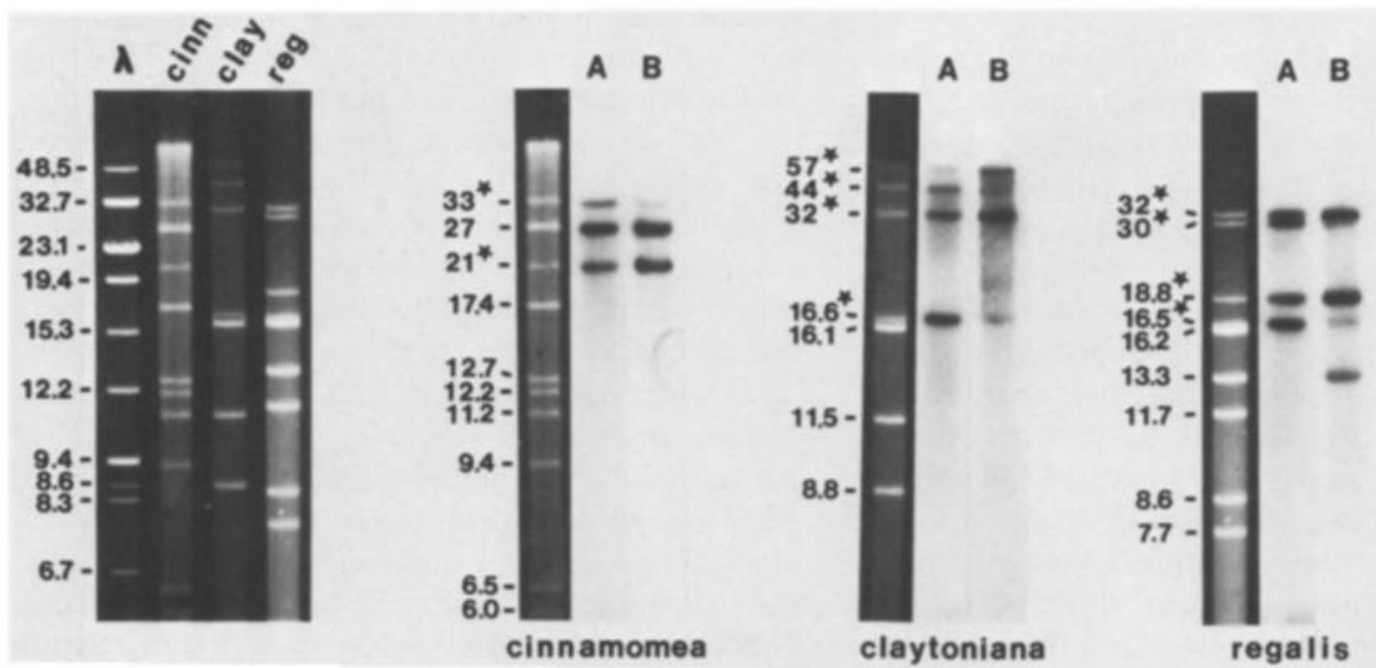

Fig. 4. Physical heterogeneity of $O s m u n d a$ cpDNAs. CpDNAs from $O$. cinnamomea, $O$. claytoniana, and $O$. regalis were digested with PstI, SacI, and SacI, respectively, and the resulting fragments were separated by electrophoresis on a $0.5 \%$ agarose gel run for $40 \mathrm{~h}$ (until the bromophenol blue tracking dye had run $20 \mathrm{~cm}$ ). The fragments were transferred to filters and subjected to hybridization with ${ }^{32} \mathrm{P}$-labelled clones containing an inverted repeat-specific fragment from mung bean (lanes marked $A$ ) and a largely small single copy region-specific fragment from $O$. regalis (lanes marked $B$ ). The exact positions in the Osmunda genomes of the sequences homologous to these clones are shown in Fig. 5 (also see text). Stars indicate half stoichiometry bands (see text). Size markers (lane marked 2) include intact lambda phage DNA $(48.5 \mathrm{~kb})$, lambda Sall fragments $(32.7 \mathrm{~kb}, 15.3 \mathrm{~kb})$, lambda HindIII fragments $(23.1 \mathrm{~kb}, 9.4 \mathrm{~kb}$, $6.7 \mathrm{~kb})$, and lambda SmaI fragments $(19.4 \mathrm{~kb}, 12.2 \mathrm{~kb}, 8.6 \mathrm{~kb}, 8.3 \mathrm{~kb})$. Only the portion of the gel above $6 \mathrm{~kb}$ is shown 

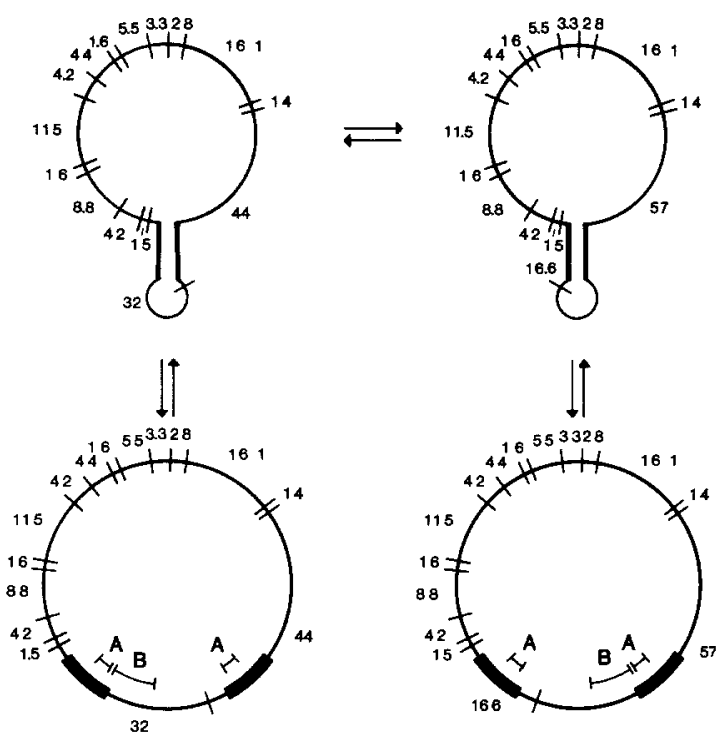

A
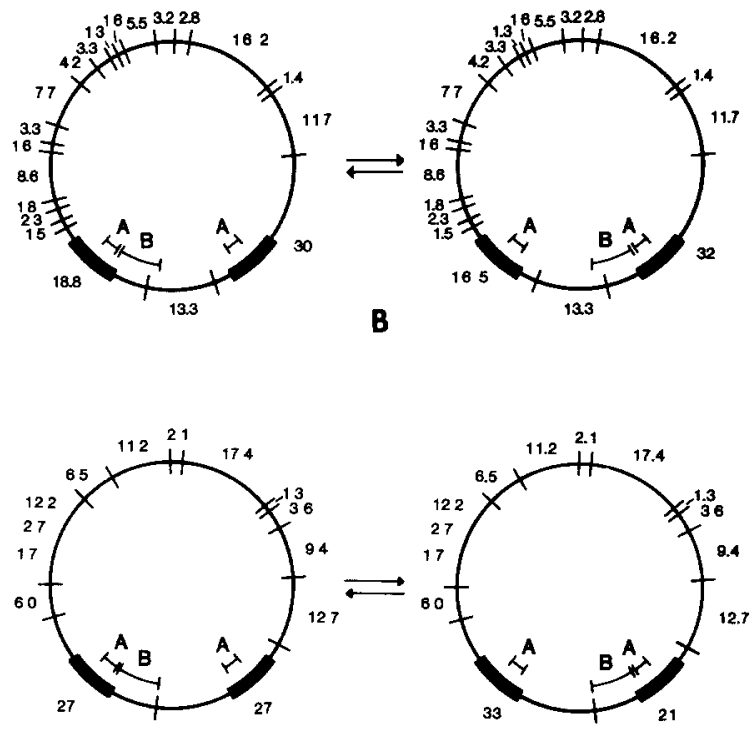

C

Fig. 5A-C. Model for isomerization of Osmunda cpDNAs via intramolecular recombination between segments of the inverted repeat. A Bottom, SacI restriction maps of the two inversion isomers of cpDNA from $O$. claytoniana. These two molecules differ only with respect to the relative orientation of their single copy DNA regions. Top, Dumbbell-shaped molecules postulated to occur as intermediates in the interconversion, via reciprocal recombination within intramolecularly paired inverted repeat segments, of the two inversion isomers shown at bottom. Radial lines on the circles indicate Sacl sites and numbers give the size of the SacI fragments. Heavy black lines indicate the location of the inverted repeat. The brackets under $A$ and $B$ indicate the positions of the corresponding hybridization probes used in Fig. 4 (also see text). B Inversion isomers of cpDNA from $O$. regalis. SacI sites and fragment sizes are indicated. C Inversion isomers of cpDNA from $O$. cinnamomea. PstI sites and fragment sizes are indicated

estimate obtained with the other three mapped enzymes (Fig. 2; Palmer and Stein 1982). For each of the other two genomes there are four half-molar SacI fragments (Fig. 4). In each case, the smallest and largest halfmolar fragments and the two intermediate half-molar fragments sum to the same approximate size $(75 \mathrm{~kb}$ for $O$. claytoniana and $49 \mathrm{~kb}$ for $O$. regalis), a number equal to the genome size obtained with SacI minus the genome size obtained with the three other mapped enzymes.

The above observations suggest that each genome might exist in two different forms with opposite polarities of the single copy regions. According to this model, each of the large half-molar fragments would contain an entire inverted repeat segment (Fig. 5). To verify this interpretation, filter hybridizations were performed using two cloned fragments as probes. The first (probe "A" in Fig. 5) is a $3.5 \mathrm{~kb}$ SacI fragment that contains most of the 23S rRNA gene and part of the transcribed rDNA spacer from mung bean (Palmer and Thompson 1981). This mung bean fragment has homology only to sequences within the inverted repeat in Osmunda, for example, the only Osmunda PvuII fragment to which it hybridizes is the $4.1 \mathrm{~kb}$ fragment internal to the inverted repeat (see Fig. 2; hybridizations not shown). The second (probe " $\mathrm{B}$ ") is the $9,7 \mathrm{~kb}$ PstI fragment from $O$. regalis that was described in the preceding section and which is localized primarily within the small single copy region, although containing about one $\mathrm{kb}$ of inverted repeat sequences. The inverted repeat-specific probe, probe A, hybiidizes to all four of the large half-molar fragments of $O$. claytoniana and $O$. regalis cpDNAs, producing hybridizations signals proportional in strength to the staining intensity of the four half-molar bands (Fig. 4). The simplest interpretation of these data is that each of the four large fragments does indeed contain an entire inverted repeat segment, with one pair of fragments present in one genomic form and the other pair in its inversion isomer (Fig. 5a, b). Consistent with this interpretation, probe $B$, the largely small single copy-specific fragment, also hybridizes to all four of the half-molar fragments in both genomes, but much more strongly to one of the two half-molar fragments from each of the two predicted inversion forms than to the other (Fig. 4; also see Fig. 5a, b). The weak hybridizations to the two other half-molar fragments can be accounted for by crosshybridization with the inverted repeat portion of probe B.

The inverted repeat probe hybridizes more or less equally strongly to the two large half-molar fragments of $O$. cinnamomea cpDNA (Fig. 4). In addition, it hybridizes about twice as strongly to the unimolar fragment of $27 \mathrm{~kb}$. We interpret this to mean that the $33 \mathrm{~kb}$ and $21 \mathrm{~kb}$ fragments contain the inverted repeats of one ge- 
nomic form, and that the $27 \mathrm{~kb}$ fragment is composed of two different comigrating half-molar fragments that reside on the inversion isomer of the first molecule (Fig. 5c). Consistent with this interpretation, probe B hybridizes approximately equally strongly to the halfmolar $21 \mathrm{~kb}$ fragment as to the unimolar $27 \mathrm{~kb}$ fragment (the putative doublet of half-molar fragments) (Fig. 4). The weak hybridization to the $33 \mathrm{~kb}$ half-molar fragment derives from the inverted repeat portion of probe B.

\section{Discussion}

Although separated by at least 70 million years of evolution (Miller 1967, 1971; Stein et al. 1979), these three Osmunda species possess chloroplast genomes that are remarkably similar in size and organization. The three fern cpDNAs have the same linear sequence order, i.e. they have not sustained any major inversions and transpositions since their divergence from a common ancestral genome. Moreover, they are almost identical in size, differing only by a number of small deletions/additions of less than $400 \mathrm{bp}$ in size. In both these respects, cpDNA appears to be evolving at least as slowly in the fern genus $O s m u n d a$ as it does among the majority of angiosperms (see Introduction).

Since chloroplast genome structure is so invariant both in Osmunda and also among most angiosperms one might predict a high degree of structural similarity between the genomes of the two groups. Indeed, we earlier noted similarities between angiosperm cpDNA and that of $O$. cinnamomea in overall size, presence and position of the rRNA-encoding inverted repeat, and location of four protein genes (Palmer and Stein 1982). In the accompanying paper, we now extend these comparisons and show that at a high level of resolution the $O$. cinnamomea chloroplast genome is essentially colinear with that of the ancestral angiosperm (Palmer and Stein 1986). In other words, the extremely conservative mode of structural evolution of cpDNA in Osmunda and in most groups of flowering plants allows one to derive a basic chloroplast genome organization that traces back to the common ancestor of vascular plants, if not all land plants.

The absence of any detectable dispersed repeated sequences in the $50 \%$ of the Osmunda chloroplast genome assayed by our cpDNA clones is one more feature in which the Osmunda genome resembles that of most angiosperms. Self-hybridizations using cloned probes have failed to detect any dispersed repeats in cpDNAs from eight angiosperms (Palmer and Thompson 1981, 1982; Coates and Cullis 1982; Ko et al. 1983; Spielmann et al. 1983; de Heij et al. 1983; Fluhr et al. 1983; J. Palmer, J. Aldrich and W. Thompson, unpublished data).
It is interesting to note, however, that those cpDNAs from several species of Chlamydomonas and from the anigosperms Trifolium subterraneum and Pelargonium hortorum - that do contain dispersed repeats are also those which show evidence of extensive and perhaps recent rearrangement (Rochaix 1978; Gelvin and Howell 1979; Lemieux and Lemieux 1985; Lemieux et al. 1985a; Palmer et al. 1985b; J. Palmer, B. Osorio and W. Thompson, unpublished data; J. Palmer, J. Nugent and L. Herbon, unpublished data). Whether these rearrangements are in any way directly related to the presence of these dispersed repeats, and conversely, whether the extreme stability of the genome in Osmunda and in most angiosperms results from the absence of repeated sequences, await further study.

Our studies add the three species of Osmunda to the list of plants for which cpDNA "flip-flip" heterogeneity has been demonstrated. In addition to the three ferns, these include the angiosperms Phaseolus vulgaris and Glycine max (Palmer 1983; Palmer et al. 1984; Mubumbila et al. 1983), the green alga Chlamydomonas reinhardtii (Aldrich et al. 1985; Palmer et al. 1985b), and Cyanophora paradoxa (Bohnert and Loffelhardt 1982), an alga of uncertain taxonomic placement. It therefore seems likely that all chloroplast genomes which contain a typical inverted repeat exist as an equimolar mixture of two inversion isomers. Yet, in spite of the presence of this recombinational dualism in chloroplast genomes spanning hundreds of millions of years of evolution, we presently have little idea as to how this recombination occurs (Palmer et al. 1985b), or what, if any, function it may serve.

Acknowledgements. We are grateful to V. James and G. Bennett for construction of the Osmunda cpDNA clone banks, and to M. Zolan for critical reading of the manuscript. This research was partially funded by a William and Flora Hewlett Foundation grant of Research Corporation to D. B. Stein, by NSF grant BSR-8415934 to J. D. Palmer, by NSF grant PCM-8109795 to W. F. Thompson, and by funds from the Carnegie Institution of Washington.

\section{References}

Aldrich J, Cherny B, Merlin E, Williams C, Mets L (1985) Curr Genet 9:233-238

Birnboim HC, Doly J (1979) Nucleic Acids Res 7:1513-1523

Bohnert HJ, Loffelhardt W (1982) FEBS Lett 150:403-406

Bohnert HJ, Crouse EJ, Schmitt JM (1982) Encycl Plant Physiol $14 \mathrm{~B}: 475-530$

Bowman CM, Bonnard G, Dyer TA (1983) Theor Appl Genet $65: 247-262$

Coates D, Cullis CA (1982) Plant Mol Biol 1:183-189

Crouse EJ, Schmitt JM, Bohnert HJ (1985) Plant Mol Biol Rep 3:43-89

De Heij HT, Lustig H, Moeskops DJM, Bovenberg WA, Bisanz C, Groot GSP (1983) Curr Genet 7:1-6 
Fluhr R, Edelman M (1981) Nucleic Acids Res 9:6841-6853

Fluhr R, Fromm H, Edelman M (1983) Gene 25:271-280

Gelvin SB, Howell SH (1979) Mol Gen Genet 173:315-322

Gillham NW, Boynton JE, Harris EH (1985) In: Cavalier-Smith T (ed) DNA and evolution: natural selection and genome size. Wiley, New York, pp 299-351

Gordon KHJ, Crouse EJ, Bohnert HJ, Herrmann RG (1982) Theor Appl Genet 61:373-384

Herrmann RG, Palta HK, Kowallik (1980) Planta 148:319-327

Herrmann RG, Westhoff P, Alt J, Winter P, Tittgen J, Bisanz C, Sears BB, Nelson N, Hurt E, Hauska G, Viebrock A, Sebald W (1983) In: Ciferri O, Dure L III (eds) Structure and function of plant genomes. Plenum Press, New York, pp 143-153

Ko K, Strauss NA, Williams JP (1983) Curr Genet 7:255-263

Lemieux B, Lemieux C (1985) Curr Genet 10:213-219

Lemieux C, Turmel M, Lee RW, Bellemare G (1985a) Plant Mol Biol 5:77-84

Lemieux C, Turmel M, Seligy VL, Lee RW (1985b) Curr Genet 9:139-145

Miller CN (1967) Contrib Mus Paleontol Univ Mich 21:139-203

Miller CN (1971) Contrib Mus Paleontol Univ Mich 23:105-169

Mubumbila M, Gordon KHJ, Crouse EJ, Burkard G, Weil JH (1983) Gene 21:257-266

Mubumbila M, Crouse EJ, Weil JH (1984) Curr Genet 8:379385

Mubumbila M, Bowman CM, Droog F, Dyer T, Kuntz M, Weil JH (1985) Plant Mol Biol 4:315-320

Ohyama K, Yamano Y, Fukuzawa H, Komano T, Yamagishi H, Fujimoto S, Sugiura M (1983) Mol Gen Genet 189:1-9

Palmer JD (1982) Nucleic Acids Res 10:1593-1605

Palmer JD (1983) Nature (London) 301:92-93

Palmer JD (1985a) Annu Rev Genet 19:325-354

Palmer JD (1985b) In: MacIntyre RJ (ed) Monographs in evolutionary biology: molecular evolutionary genetics. Plenum Press, New York, pp 131-240

Palmer JD (1985c) Methods Enyzmol 118:167-186

Palmer JD, Stein DB (1982) Curr Genet 5:165-170

Palmer JD, Stein DB (1986) Curr Genet 10:823-833
Palmer JD, Thompson WF (1981) Proc Natl Acad Sci USA 78: 5533-5537

Palmer JD, Thompson WF (1982) Cell 29:537-550

Palmer JD, Shields CR, Cohen DB, Orton TJ (1983a) Theor Appl Genet $65: 181-189$

Palmer JD, Singh GP, Pillay DTN (1983b) Mol Gen Genet 190: 13-19

Palmer JD, Osorio B, Watson JC, Edwards H, Dodd J, Thompson WF (1984) In: Thornber JP, Staehelin LA, Hallick RB (eds) Biosynthesis of the photosynthetic apparatus: molecular biology, development and regulation. Liss, (UCLA Symposia on Molecular and Cellular Biology, New series, vol 14) New York, pp 273-283

Palmer JD, Jorgensen RA, Thompson WF (1985a) Genetics 109: $195-213$

Palmer JD, Boynton JE, Gillham NW, Harris EH (1985b) In: Steinback KE, Bonitz S, Arntzen CJ, Bogorad L (eds) Molecular biology of the photosynthetic apparatus. Cold Spring Harbor Laboratory, Cold Spring Harbor, NY, pp 269-278

Rochaix JD (1978) J Mol Biol 126:597-617

Salts Y, Herrmann RG, Peleg N, Lavi U, Izhar S, Frankel R, Beckmann JS (1984) Theor Appl Genet 69:1-14

Spielmann A, Ortiz W, Stutz E (1983) Mol Gen Genet 190:5-12

Stein DB, Thompson WF, Belford HS (1979) J Mol Evol 13: $215-232$

Takaiwa F, Sugiura M (1982) Nucleic Acids Res 10:2665-2676

Vieira J, Messing J (1982) Gene 19:257-268

Whitfeld PR, Bottomley W (1983) Annu Rev Plant Physiol 34: $279-310$

Zurawski G, Clegg MT, Brown AHD (1984) Genetics 106:735749

Communicated by K. P. VanWinkle-Swift

Received December 3, 1985 / March 3, 1986 